\title{
Globe
}

Revue internationale d'études québécoises

\section{Alessandra Ferraro, Écriture migrante et translinguisme au Québec, Venise, La Toletta, 2014}

\section{Gilles Dupuis}

Volume 17, numéro 2, 2014

URI : https://id.erudit.org/iderudit/1036253ar

DOI : https://doi.org/10.7202/1036253ar

Aller au sommaire du numéro

Éditeur(s)

Globe, Revue internationale d'études québécoises

ISSN

1481-5869 (imprimé)

1923-8231 (numérique)

Découvrir la revue

Citer ce compte rendu

Dupuis, G. (2014). Compte rendu de [Alessandra Ferraro, Écriture migrante et translinguisme au Québec, Venise, La Toletta, 2014]. Globe, 17(2), 236-238.

https://doi.org/10.7202/1036253ar d'utilisation que vous pouvez consulter en ligne.

https://apropos.erudit.org/fr/usagers/politique-dutilisation/ 
subtilité, dont on peut supposer qu'elle deviendra une référence incontestable pour toute personne s'intéressant à la littérature des femmes.

Catherine Dussault Frenette

Université de Sherbrooke

\section{Alessandra Ferraro \\ Écriture migrante et translinguisme au Québec, Venise, La Toletta, 2014.}

Dans ce court mais très dense ouvrage consacré au corpus des écritures migrantes en relation avec la dimension linguistique, composé de sept articles déjà parus (dont plusieurs ont été remaniés ou traduits en français) et de deux textes inédits, Alessandra Ferraro parvient sinon à renouveler l'approche de ce corpus, du moins à nuancer l'apport de l'écriture migrante au canon littéraire québécois, tout en éclairant ce que plusieurs travaux sur le même corpus avaient laissé dans l'ombre, notamment le rôle des filiations dans l'émergence d'une littérature migrante propre au Québec.

Après un "avant-propos" qui a le mérite de situer la question de la transculture québécoise dans le contexte plus vaste d'une "esthétique postcoloniale" (p. 13), l'ouvrage se divise en deux grandes parties. La première est consacrée au "cadre contextuel " dans lequel ont émergé les écritures migrantes au cours des années 1980, tandis que la deuxième se compose d'analyses de cas regroupées sous le titre "dynamiques textuelles". L'on passe donc logiquement de la théorie à la pratique, la théorisation du phénomène migrant préparant la voie à l'étude de quelques cas retenus en fonction du rapport qu'ils permettent d'établir entre littérature et langue d'écriture d'une part, entre transculture et translinguisme littéraire d'autre part.

Si le cadre contextuel est sans doute la partie de l'ouvrage qui surprend le moins pour qui connaît déjà le sujet, il apporte en revanche des éléments inédits ou à tout le moins méconnus pour la compréhension du phénomène global de l'écriture migrante et de sa relative nouveauté dans le panorama de la littérature québécoise contemporaine. L'un de ses apports est sans conteste la mise en lumière de la filiation souterraine qui lie, dans le chapitre consacré à "l'archéologie de la transculture ", la revue italienne 
montréalaise Quaderni culturali au magazine transculturel italo-québécois Vice Versa. La critique avait déjà insisté sur le rôle pionnier joué par la revue interculturelle Dérives (1975-1987) dans l'avènement du discours qui allait devenir par la suite "transculturel », mais elle avait peu tenu compte du rôle également de précurseur joué par les Quaderni culturali (1980-1982), sans doute parce que cet organe de diffusion avait été perçu, en raison du choix de la langue destiné à la communauté italienne, comme une revue "ethnique ". Il n'en demeure pas moins l'ancêtre direct du magazine trilingue auquel il donnera le jour dans son dernier numéro paru en 1982, comme le rappelle fort à propos Alessandra Ferraro. Il fallait sans doute une chercheuse italienne pour s'intéresser de près à cette revue éphémère (mais à la postérité durable) et pour révéler, lectures et traductions à l'appui, sa réelle fonction de chaînon manquant dans la transmission de la transculture au Québec.

L'autre apport original de cet ouvrage réside dans sa problématique, qui se concentre autour des liens qu'entretient l'écriture migrante avec la question du translinguisme. En faisant jouer les concepts de l'interdiscursivité (Bakhtine), de l'intertextualité (Kristeva) et de l'hypertextualité (Genette), mais aussi les concepts bakhtiniens d'hétéroglossie, d'hétérophonie et d'hétérologie, l'auteure entend montrer en quoi les écritures migrantes ne sont pas seulement transculturelles, mais aussi nécessairement translinguistiques. Que la langue d'écriture soit uniquement le français, comme c'est le cas (à quelques exceptions près) pour les écrivains Monique Bosco, Régine Robin, Carole David et Wajdi Mouawad, ou qu'elle soit multiple, par exemple chez Marco Micone et encore plus chez Antonio D'Alfonso, elle est toujours habitée par une autre langue, pour ne pas dire la langue de l'Autre, ce à quoi renvoie le concept bakhtinien de dialogisme (ou polyphonie), bien plus que la notion de plurilinguisme qui peut à la limite être monologique. Avant même que soit considéré l'exercice particulier de l'autotraduction, pratiquée par les deux auteurs d'origine italienne, la thèse défendue par Ferraro nous convainc que la langue de l'écrivain migrant est toujours déjà habitée par celle d'un ou d'une autre (parent, ami, amante), à la fois proche et lointaine, étrangement familière ou familièrement inquiétante, selon le double sens du mot allemand - et désormais freudien - Unheimlich.

L'exemple de l'écrivain italo-québécois Antonio D'Alfonso, à qui sont d'ailleurs consacrées deux des cinq analyses de cas qui servent à illustrer le cadre théorique de l'ouvrage, est à ce titre emblématique : tiraillé entre deux grandes cultures (italienne et nord-américaine) et partagé entre trois ou quatre langues d'origine (le français, l'anglais, l'italien et le dialecte molisan), il est l'écrivain transculturel et translinguistique par excellence, d'autant qu'il 
constitue avec Marco Micone, bien que dans un tout autre esprit, l'auteur du corpus étudié qui se livre le plus à l'activité féconde de l'autotraduction. À son sujet, l'auteure met bien en évidence ce que la notion d'italicité a de spécifique et de pertinent par rapport au concept plus largement partagé de transculture dans sa communauté d'origine. Bien qu'elle semble limitée a priori aux collectivités italiennes expatriées, dont la communauté italoquébécoise, la théorie dalfonsienne d'une culture "italique» (p. 87) peut servir d'exemple à tout "migrant", de l'extérieur comme de l'intérieur. Antonio D'Alfonso est d'ailleurs le seul écrivain du corpus (à part Carole David) à n'avoir pas été d'abord un immigrant avant d'acquiescer pleinement à la condition migrante. Si, comme l'avance Ferraro, «[l] a figure du Phénix [est une] représentation emblématique du créateur migrant» (p. 62), l'exemple protéiforme de D'Alfonso nous invite à recourir à celle du caméléon pour mieux caractériser «la culture métissée du migrant» (p. 62).

Au final, l'ouvrage d'Alessandra Ferraro se signale à notre attention à plus d'un titre: originalité de l'angle d'approche, qui permet de présenter sous un jour nouveau un corpus bien balisé par la critique, pertinence du cadre théorique et heuristique de la recherche, minutie et attention soutenue dans l'analyse des œuvres choisies. D'autres cas exemplaires auraient pu être convoqués, mais ceux retenus sont suffisamment variés pour offrir un très bon aperçu de la redéfinition du canon littéraire opérée par l'écriture migrante «translinguistique». Un souci éditorial plus grand aurait certes pu être apporté à la révision finale du manuscrit. Cette lacune n'enlève rien à la valeur intrinsèque de l'ouvrage, qui sera consulté avec profit par tout chercheur qui s'intéresse au phénomène de l'écriture migrante au Québec, en relation (ou non) avec la question du translinguisme.

Gilles Dupuis

Université de Montréal 